\title{
Null Space Based Robot Compliant Control with Prescribed Motion Performance
}

\author{
Yiming Jiang \\ College of Automation Science \\ and Engineering, \\ South China University of Technology \\ Guangzhou 510640, China.
}

\author{
Chenguang Yang \\ College of Automation Science \\ and Engineering, \\ South China University of Technology \\ Guangzhou 510640, China. \\ Email: cyang@ieee.org
}

\author{
Zhaojie Ju \\ School of Computing, \\ University of Portsmouth \\ Portsmouth PO13HE, UK.
}

\author{
Min Wang \\ College of Automation Science and Engineering, \\ South China University of Technology \\ Guangzhou 510640, China.
}

\author{
Honghai Liu \\ School of Computing, \\ University of Portsmouth \\ Portsmouth PO13HE, UK.
}

\begin{abstract}
In this paper, a null space controller is proposed to achieve a compliant motion behaviour of a redundant manipulator for a safety human-robot interaction. The robot endeffector is controlled using a prescribed performance controller, such that the transient tracking performance of the main task is guaranteed. The compliant behaviour of the robot motion is ensured by using a null space control law, by which the external torque acting on the main task can be projected to a null space and the effects of the external force can be reduced. The external torque is estimated and canceled by using an adaptive disturbance observer. Lyapunov synthesis illustrates the asymptotic stability of the robot system while simulation studies based on a 3-DOF planner robot show the effectiveness of the proposed control algorithm.
\end{abstract}

Keywords-human-robot interaction; redundant robot; null space; output constraint; adaptive disturbance observer

\section{INTRODUCTION}

Recent developments in human-robot interactions have aroused an expectation that the robots can serve as coworkers in a human surrounded environment. Different to traditional industrial robots, which focus on the motion precision with a high stiffness, the cooperative robots are often designed with compliance and flexibility to guarantee safety - the most important issue in the physical human robot interaction. While many delicate mechanisms such as variable stiffness actuators [1] and series elastic actuators [2] have been designed to improve the flexibility of the robots, there is still a great challenge of designing an efficient robot control scheme to meet a demand of human-robot physically interacted in a complex environment.

To ensure a safe human-robot collaboration, various control frameworks have been designed. Collision free controllers have been designed for avoiding possible collisions to the obstacles and the nearby human. In [3], the automatic obstacle avoidance behaviour was achieved by using a shared controller integrating with visual camera information and robot kinematics, where the robot body are able to avoid an obstacle while guarantee the stability of the teleoperated manipulation. A joint acceleration level collision avoidance controller was proposed for a redundant robot using a minimum acceleration norm and an inequality-based criterion [4]. In the afromentioned approaches, the controllers were designed to immediately move the robots away from the collision points. However, it would be more desirable to perform a compliant robot control behaviour when the robot interacts or even contacts with human, regarding with the demand of the human-robot coexistent tasks.

For a redundant manipulators, namely, the degree of freedom (DOF) of the joint space is larger than the DOF in the task space, the null space impedance control is useful to achieve a compliance control performance. When a external interaction is exerted on robot body, this compliant behaviour was realized by projecting the interaction forces to the null space of task space without affect the main mission [5], [6]. It is well known that impedance control approaches are helpful to realized an actively robotic compliant control. The impedance controllers were usually designed for controlling the robot end-effector interacted with the environment. However, it can also be employed for the joint space to improve the compliance of the robot body. In [7], a passivity-based impedance framework was designed for a DLR flexible joint robots with the compliant behaviour using the singular perturbation method. A multipriority impedance control framework was proposed by selecting orders of the impedance priority in the task space [8]. By combining the null space control and the impedance control, a null space impedance controller was designed for an acceleration level multipriority control [9].

It should be noted that the external toque exerted on the 
robot is not easy to measure. In order to guarantee the compliant impedance control performance without interfering the main task, the external force need to be estimated and properly canceled. In [6], an adaptive observer was employed to reduce the task space error caused by the exerted external forces. A singularity free orientation compliant controller was developed without using torque sensors information based on a disturbance observer control approach [10]. In the aforementioned works, however, the transient tracking performance of the task space control was not regularly guaranteed. In some applications, it is desirable to achieve a predefined tracking performance. Therefore, in this paper, the output constraint control is used by posing constraints on the endeffector position to regulate the system tracking performance. The guaranteed performance control of a five-bar robot was investigated in [12], where an error transformation algorithm was developed to ensure the control performance in both stable and transient stages.A proper barrier Lyapunov function based control scheme was developed for the robot motion control with enhanced transient tracking performance [11].

By the above discussion, in this paper, a null space based robot controller is developed to guarantee both the transient tracking control performance and the compliant motion control behaviour. The prescribed transient tracking performance is ensured by using an error transformation based task space controller, and the compliant behaviour is achieved by using the null space dynamics control algorithm. In addition, an adaptive disturbance observer is designed to cancelled the effect of the external torque. The system stability is regularly proved based on the Lyapunov theorem.

\section{PRELIMINARIES}

\section{A. System Description}

The dynamics of a n-link robot manipulator can be described as

$$
M(q) \ddot{q}+C(q, \dot{q}) \dot{q}+G(q)+\tau_{d}=\tau
$$

where $q$ denotes the joint angles, $M(q)$ is an inertia/mass matrix, $C(q, \dot{q})$ is a Coriolis/centrifugal matrix, $G(q)$ is a gravity vector, $\tau$ is the joint control torque to be designed and $\tau_{d}$ is the external torque exerted on the joint which may be caused by the interaction of environment or unmodelled disturbances.

The joint space impedance control can be described as

$$
M_{d}\left(\ddot{q}_{d}-\ddot{q}\right)+B_{d}\left(\dot{q}_{d}-\dot{q}\right)+K_{d}\left(q_{d}-q\right)=\tau_{d}
$$

where $q_{d}$ denotes the desired joint trajectory, and $M_{d} \in$ $\mathbb{R}^{n \times n}, B_{d} \in \mathbb{R}^{n \times n}$ and $K_{d} \in \mathbb{R}^{n \times n}$ are the desired inertia matrix, damping matrix and stiffness matrix, respectively, which are chosen positive definite.

Considering a n-degree of freedom (DOF) redundant robot manipulator, the relationship between the end-effector velocity and the joints velocity can be described using the following kinematic equations,

$$
\dot{x}=J(q) \dot{q}
$$

where $\dot{x}$ is a vector of the task space velocity and $J(q)$ denotes the Jacobian matrix with respect to $q$.

For a redundant manipulator, namely, the DOF of the joint space is larger than the DOF of the task space, the Jacobian matrix is not square since the line number is smaller than the column number. This means that, for a given task space velocity $\dot{x}$, the inverse solution of $\dot{q}$ may not be unique. Therefore, it is possible to control the task space and the contact space simultaneously. This can be fulfilled by using a general inverse solution called null space control, which is described as below

$$
\dot{q}=J^{\dagger} \dot{x}+N \dot{q}_{m}
$$

where $J^{\dagger}(q)$ is an inverse of $J(q)$ and $N$ is a null space matrix which can be given by

$$
N=I-J^{\dagger} J
$$

\section{B. Joint Space Decomposition}

In order to describe the motions of a redundant manipulator, a joint space decomposition algorithm is employed [13]. By using an auxiliary matrix $Z(q)$ and an auxiliary vector $\lambda$, $\dot{q}$ can be formulated as

$$
\dot{q}=N \dot{q}_{m}=Z(q) \lambda
$$

where $Z(q)$ satisfies that $J(q) Z(q)=\mathbf{0}$, and $\lambda$ is a $r$ dimensional velocity vector. A general solution of $\lambda$ can be obtained by using a left inertia-weighted generalized inverse as [14]

$$
\lambda=Z(q)^{\#} \dot{q}=\left(Z(q)^{T} M(q) Z(q)\right)^{-1} Z(q)^{T} M(q)
$$

such that

$$
\left(\begin{array}{c}
\dot{x} \\
\lambda
\end{array}\right)=J_{C}(q) \dot{q}=\left(\begin{array}{c}
J(q) \\
Z^{\#}(q)
\end{array}\right) \dot{q}
$$

with $J_{C}(q)$ being a non-singular matrix as $J$ is full rank. The inverse of $J_{C}(q)$ can be calculated by

$$
J_{C}^{-1}(q)=\left[J^{\#}(q) \quad Z(q)\right]
$$

where $J^{\#}(q)=M^{-1} J^{T}\left(J M^{-1} J^{T}\right)^{-1}$.

Then, in terms of (8) and (9), the decomposition of joint velocity can be achieved as

$$
\dot{q}=J^{\#} \dot{x}+Z \lambda
$$

\section{Acceleration Control}

To control the robot manipulator in both the task space and joint space, the following control law is chosen for the robot manipulator,

$$
\tau=M(q) \ddot{q}_{c}+C(q, \dot{q}) \dot{q}+G(q)+\tau_{d}
$$

and the joint command $\ddot{q}_{c}$ can be chosen as

$$
\ddot{q}_{c}=\ddot{q}_{d}+M_{d}^{-1}\left(B_{d} \dot{\tilde{q}}+K_{d} \tilde{q}-\tau_{d}\right)
$$

where $\tilde{q}=q_{d}-q$. By selecting the inertia matrix $M_{d}$ as $M_{d}=M(q)$, and taking the combination of (11) and (12), 


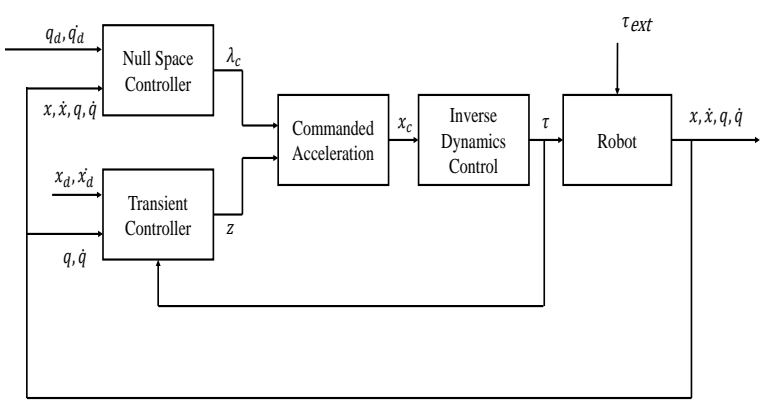

Fig. 1. An overlook of the proposed controller

(11) can be rewritten as

$$
\tau=M(q) \ddot{q}_{d}+B_{d} \dot{\tilde{q}}+K_{d} \tilde{q}+C(q, \dot{q}) \dot{q}+G(q)
$$

In terms of (11), (13) and (4), and assume that the external torque $\tau_{d}$ can be measured, then $\ddot{q}_{c}$ can be designed as,

$$
\ddot{q}_{c}=J^{\dagger}\left(\ddot{x}_{c}-\dot{J} \dot{q}\right)+N\left(\ddot{q}_{d}+M_{d}^{-1}\left(B_{d} \dot{\tilde{q}}+K_{d} \tilde{q}-\tau_{d}\right)\right)
$$

where $x_{c}$ is the commanded task space acceleration. Then the closed-loop robot system can be rewritten to

$$
\begin{gathered}
\ddot{x}=\ddot{x}_{c}-J M^{-1} \tau_{d} \\
\left.\left.N\left(\ddot{\tilde{q}}+M_{d}^{-1}\left(B_{d} \dot{\tilde{q}}+K_{d} \tilde{q}\right)\right)-M^{-1} \tau_{d}\right)\right)=0
\end{gathered}
$$

Based on (15) and (16), the task space behaviour is decoupled from the null space dynamics. In other words, we can assign the task space to the end-effector coordinate and then achieve the joint motion regulation in the null space of the task space. In this manner, we can control not only the end-effector point of the robot, but also the motion of robot body point.

Based on (8) and (10), we have the following kinematic level command acceleration

$$
\ddot{q}_{c}=Z(q)\left(\dot{\lambda}_{c}-\dot{Z}^{\#} \dot{q}\right)+J^{\#}\left(\ddot{x}_{c}-\dot{J} \dot{q}\right)
$$

where $\dot{\lambda}_{c}$ is the virtual control inputs of the null space accelerations.

Having in mind that $\dot{\lambda}=\dot{Z}^{\#} \dot{q}+Z^{\#} \ddot{q}$, we have the closedloop null space dynamic equation as below

$$
\dot{\lambda}=\dot{\lambda}_{c}-Z^{\#} M^{-1} \tau_{d}
$$

\section{Prescribed Performance Control}

A technique called prescribed performance control (PPC) is introduced to regulate the control performance of the robot in the task space. Define a tracking error as $e=x-x_{d}$, where $x$ is the task space trajectory and $x_{d}$ is the desired tracking trajectory.

The following functions are introduced for the prescribed motion performance,

$$
\rho=\left(\rho_{0}-\rho_{\infty}\right) e^{-a t}+\rho_{\infty}
$$

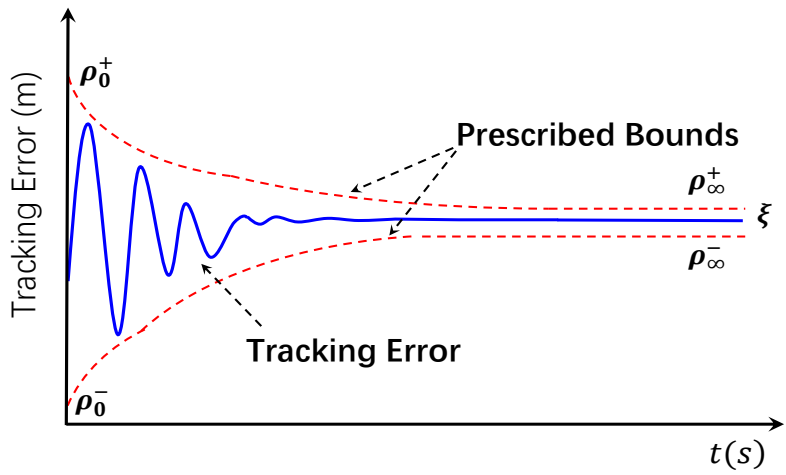

Fig. 2. The prescribed performance control

$$
R_{i}(x)= \begin{cases}\left(\frac{e^{x}-\delta}{1+e^{x}}\right) & \text { if } \quad e \geq 0 \\ \left(\frac{\delta e^{x}-1}{1+e^{x}}\right) & \text { if } \quad e<0\end{cases}
$$

where $\rho_{0}, \rho_{\infty}, a$ and $\delta$ are specified parameters to regulate the transient control performance. Based on (19) and (20), an error transformation function can be designed as below [12]

$$
e_{i}=\rho(t) R_{i}\left(\xi_{i}(t)\right)
$$

where $\xi_{i}$ is the transformed error. According to the above error transformation design, we have that $-\delta<R_{i}<1$ when $e \geq 0,<-1<R_{i}<\delta$ when $e<0$. Then we can derive that, if $\xi_{i}$ is bounded, then $-\delta \rho<e_{i}<\rho(e>0)$ and $-\rho<e<\delta \rho(e<0)$. This means that $\rho$ can be used to specify the upper and lower bounded of the tracking error $e$ when $\xi_{i}$ is bounded, as show in Fig. 2.

According to (20) and (21), the solution of $\xi_{i}$ can be obtained as

$$
\xi_{i}(t)=L_{i}\left(e_{i}(t) / \rho\right)
$$

where

$$
L_{i}(x)= \begin{cases}\left(\ln \frac{x+\delta}{1-x}\right) & \text { if } \quad e \geq 0 \\ \left.\ln \frac{x+1}{\delta-x}\right) & \text { if } \quad e<0\end{cases}
$$

The time derivative of (22) is given as

$$
\dot{\xi}_{i}(t)=\frac{\dot{L}_{i}\left(R_{i}\left(\xi_{i}(t)\right)\right)}{\rho^{2}(t)}(\rho(t) \dot{e}-\dot{\rho}(t) e)
$$

with $\dot{L}(x)=\frac{1+\delta}{(x+\delta)(1-x)}(e \geq 0)$ and $\dot{L}(x)=\frac{1+\delta}{(x+1)(\delta-x)}(e<$ $0)$. Based on the above mentioned analysis, the control objective is to guarantee the boundedness of $\xi_{i}$, such that the prescribed tracking performance can be achieved.

\section{Control Design}

The control objective is to guarantee the motion control performance of the end-effector for tracking a desired trajectory while allowing the robot body to perform a compliant behaviour in the null space. An overlook of the proposed controller is depicted as shown in Fig. 1 


\section{A. Control design for the main task}

Define a residual error of the robot tracking as

$$
z=\dot{x}-v_{d}
$$

where $v_{d}$ is an auxiliary controller to be design later.

Considering the task dynamics (15), in order to track the desired trajectory $x_{d}$ with prescribed performance, the following acceleration control law is designed,

$$
\ddot{x}_{c}=\dot{v}_{d}-M_{x}^{-1}\left(\left(C_{x}+K_{p}\right) z-\frac{\dot{L} \xi}{\rho}\right)+J^{\# T} \hat{\tau}_{d}
$$

where $K_{p}$ is a positive definite matrix, $\hat{\tau}_{d}$ is the estimation of the external torque which will be specified later, $M_{x}=$ $\left(J M^{-1} J^{T}\right)^{-1}$ is the inertial matrix in task space, and $C_{x}$ is the task space Coriolis and centrifugal matrix.

The combination of (26) and (15) yields

$$
\ddot{x}=\dot{v}_{d}-M_{x}^{-1}\left(\left(C_{x}+K_{p}\right) z-\frac{\dot{L} \xi}{\rho}\right)+J^{\# T} \hat{\tau}_{d}-J M^{-1} \tau_{d}
$$

Let us define $v_{d i}$ as $v_{d i}=k_{1} \rho \xi_{i}+\dot{x}_{d}+e \dot{\rho} / \rho(t)$, where $k_{1}$ is the positive control gain. Thus, the boundedness of the $\xi$ can be achieved as long as $z$ converges to zero. Then, (24) can be written by taking the definition of $v_{d}=\left[v_{d 1}, \cdots, v_{d m}\right]$ into consideration as

$$
\dot{\xi}_{i}(t)=-k_{1} \dot{L}\left(R_{i}\left(\xi_{i}(t)\right)\right) \xi_{i}+\frac{\dot{L}\left(R_{i}\left(\xi_{i}(t)\right)\right)}{\rho(t)} z_{i}
$$

Multiplying $M_{x}$ on both sides of (27), we have

$$
M_{x} \ddot{x}+J^{\# T} \tau_{d}=M_{x} \dot{v}_{d}-\left(C_{x}+K_{p}\right) z+\frac{\dot{L} \xi}{\rho}+J^{\# T} \hat{\tau}_{d}
$$

where the fact $J^{\#}(q)=M^{-1} J^{T}\left(J M^{-1} J^{T}\right)^{-1}$ was employed. Then, the closed-loop task space dynamics can be described as

$$
M_{x} \dot{z}=-\left(C_{x}+K_{p}\right) z+\frac{\dot{L} \xi}{\rho}+J^{\# T} \tilde{\tau}_{d}
$$

where $\tilde{\tau}_{d}=\hat{\tau}_{d}-\tau_{d}$.

Based on the adaptive disturbance observer in [16], the adaptive law for the estimated external torque $\hat{\tau}_{d}$ is designed as

$$
\dot{\hat{\tau}}_{d}=-\Gamma^{-1} J^{\#} z
$$

where $\Gamma$ is a designed positive definite matrix.

\section{B. Control design for null space task}

Generally speaking, the null-space velocity $\lambda$ can not be integrated, therefore, we can not define a null space position error for the system. Alternative, a null space command acceleration can be defined based on (18) and the joint space error as

$$
\dot{\lambda}=\dot{\lambda}_{d}+M_{\lambda}^{-1}\left(\left(C_{\lambda}+K_{\lambda}\right) \tilde{\lambda}+Z^{T} K_{v} \tilde{q}\right)
$$

where $K_{\lambda}$ and $K_{v}$ are positive-definite and symmetric matrix to be designed, respectively. And $M_{\lambda}^{-1}=Z^{T} M Z$ is the inertia matrix of the null space, while $C_{\lambda}$ is the null space Coriolis and centrifugal matrix.
Based on (18) and (32), the closed-loop null space dynamics can be formulated as

$$
M_{\lambda} \dot{\tilde{\lambda}}+\left(C_{\lambda}+K_{\lambda}\right) \tilde{\lambda}+Z^{T} K_{v} \tilde{q}=Z^{T} \tau_{d}
$$

\section{Stability ANALYsis}

Theorem 1: Consider a redundant manipulator with dynamics described by (1), the task space closed-loop dynamics in (15) and null space closed-loop dynamics (18), the control laws (26) and (7) can guarantee the robot to track the desired trajectory with a compliant null space behaviour, while $\xi, z$ and $v$ can converge to zero asymptotically and the prescribed motion constraint is never violated. In addition, the estimated torque is bounded and the closed loop system is stable.

Proof: Let us consider the following Lyapunov function,

$$
V_{1}=\frac{1}{2} \xi^{T} \xi
$$

Differentiate (34) with respect to time and substituting (28) into it, we have

$$
\dot{V}_{1}=\frac{\xi^{T} \dot{L}(\xi) z(t)}{\rho(t)}-K_{1} \xi^{T} \dot{L}(\xi) \xi
$$

where $\dot{L}(\xi)=\operatorname{diag}\left(\dot{L}_{1}\left(R_{1}(\xi(t))\right), \cdots, \dot{L}_{m}\left(R_{m}(\xi(t))\right)\right)$.

Then, the following Lyapunov function is designed as,

$$
V_{2}=\frac{1}{2} z^{T} M_{x} z+\frac{1}{2} \tilde{\tau}_{d}^{T} \Gamma \tilde{\tau}_{d}
$$

where $\Gamma$ is a designed positive definite matrix.

Taking the time derivative of (36) yields,

$$
V_{2}=z^{T} M_{x} \dot{z}+\frac{1}{2} z^{T} \dot{M}_{x} z+\tilde{\tau}_{d}^{T} \Gamma \dot{\hat{\tau}}_{d}
$$

Substituting (30) into (37), we have

$$
\begin{aligned}
\dot{V}_{2} & =z^{T}\left(-\left(C_{x}+K_{p}\right) z+\frac{\dot{L} \xi}{\rho}+J^{\# T} \tilde{\tau}_{d}\right) \\
& +\frac{1}{2} z^{T} \dot{M}_{x} z+\tilde{\tau}_{d}^{T} \Gamma \dot{\hat{\tau}}_{d}
\end{aligned}
$$

Then, substituting (31) into (38) and considering $\dot{M}_{x}-2 C_{x}$ is a skew-symmetry matrix, we have

$$
\begin{aligned}
\dot{V}_{2} & =-z^{T} K_{p} z+J^{\# T} \tilde{\tau}_{d}-\tilde{\tau}_{d}^{T} J^{\#} z+z^{T} \frac{\dot{L} \xi}{\rho} \\
& =-z^{T} K_{p} z+z^{T} \frac{\dot{L} \xi}{\rho}
\end{aligned}
$$

Then, combining (35) and (39) yields,

$$
\begin{aligned}
\dot{V}_{1} & +\dot{V}_{2} \\
& =-z^{T} K_{p} z+z^{T} \frac{\dot{L} \xi}{\rho}+\frac{\xi^{T} \dot{L}(\xi) z(t)}{\rho(t)}-K_{1} \xi^{T} \dot{L}(\xi) \xi \\
& =-z^{T} K_{p} z-K_{1} \xi^{T} \dot{L}(\xi) \xi
\end{aligned}
$$

Noticed that the inequality $\xi^{T} \dot{L}(\xi) \xi \geq 2\|\xi(t)\|^{2} / 1+\delta$ can be derived in terms of the definition of $\dot{L}(\xi)$, then we have

$$
\dot{V}=\dot{V}_{1}+\dot{V}_{2}=-z^{T} K_{p} z-\frac{2 K_{1}\|\xi(t)\|^{2}}{1+\delta} \leq 0
$$




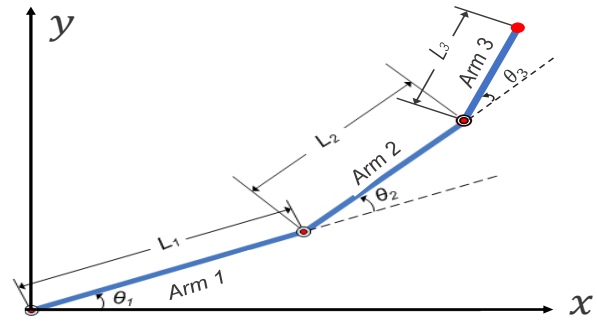

Fig. 3. The 3 DOF planner robot

According to the LaSalle's invariance principle [17], the asymptomatic stability of the closed-loop system can be proved, i.e., $z$ and $\xi$ converge to zero asymptomatically, and $\tilde{\tau_{d}}$ is uniformly ultimately bounded.

To further show the stability of the null space closed-loop system in the condition of a subset $\mathcal{C}=\left\{\tilde{q}, \tilde{\lambda}, \tilde{\tau}_{d}, \xi=0, z=\right.$ $0\}$, let us consider the Lyapunov candidate $V_{\mathcal{C}}$ as follows,

$$
V_{\mathcal{C}}=\frac{1}{2} \tilde{\lambda}^{T} M_{\lambda}(q) \tilde{\lambda}+\frac{1}{2} \tilde{q}^{T} K_{v} \tilde{q}+\frac{1}{2} \tilde{\tau}_{d}^{T} \Gamma \tilde{\tau}_{d}
$$

which is positive definite in $\mathcal{C}$. Differentiate (42) with respect to time yields,

$$
\dot{V}_{\mathcal{C}}=\tilde{\lambda}^{T} M_{\lambda}(q) \dot{\tilde{\lambda}}+\frac{1}{2} \lambda^{T} \dot{M}_{\lambda}(q) \lambda+\tilde{q}^{T} K_{v} \dot{\tilde{q}}+\tilde{\tau}_{d}^{T} \Gamma \dot{\hat{\tau}}_{d}
$$

Substituting (33) into (43), we have

$$
\begin{aligned}
\dot{V}_{\mathcal{C}} & =\tilde{\lambda}^{T}\left(\left(-\left(C_{\lambda}+K_{\lambda}\right) \tilde{\lambda}-Z^{T} K_{v} \tilde{q}+Z^{T} \tau_{d}\right)\right. \\
& +\frac{1}{2} \lambda^{T} \dot{M}_{\lambda}(q) \lambda+\tilde{q}^{T} K_{v} \dot{\tilde{q}}+\tilde{\tau}_{d}^{T} \Gamma \dot{\hat{\tau}}_{d}
\end{aligned}
$$

Since $M_{\lambda}-2 C_{\lambda}$ is a skew-symmetric matrix, and considering that $\dot{\hat{\tau}}_{d}=0$ in terms of $z=0$ and $\dot{q}=Z \lambda$, then we have

$$
\dot{V}_{\mathcal{C}}=-\tilde{\lambda}^{T} K_{\lambda} \tilde{\lambda}-\tilde{\lambda}^{T} Z^{T} \tau_{d}
$$

From the definition of $Z$, we have $Z^{T} \tau_{d}=0$. Then we can obtain that

$$
\dot{V}_{\mathcal{C}}=-\tilde{\lambda}^{T} K_{\lambda} \tilde{\lambda} \leq 0 .
$$

According to the Lasalle's theorem, the null space system in the subset $\mathcal{C}$ is asymptotically stable, and the system states converge to an invariant set defined by $\{z=0, \xi=0, \tilde{\lambda}=$ $\left.0, Z^{T} K_{v} \tilde{q}=0\right\}$.

Based on the above stability analysis, we can therefore obtain that the system states $\xi, z$ and $\lambda$ are asymptotic stable. Hence, the prescribed motion performance in the task space and compliant behaviour in the joint space can be guaranteed. This completes the proof.

\section{Simulation Study}

To demonstrate the effectiveness of the proposed algorithms, simulations are carried out based on a 3 DOF planner robot arm as shown in Fig. 3. The kinematic and inertia parameters of each link of robot are given in Table.I. The dynamics of the robot arm is described as follows [18]:

$$
M(q) \ddot{q}+C(q, \dot{q}) \dot{q}+G(q)+\tau_{d}=\tau
$$

TABLE I

RoBOTIC PARAMETERS

\begin{tabular}{llll}
\hline \hline Parameters & Link 1 & Link 2 & Link 3 \\
\hline link length $(\mathrm{m})$ & $l_{1}=1.6$ & $l_{2}=1.3$ & $l_{3}=0.7$ \\
link mass $(\mathrm{kg})$ & $m_{1}=10$ & $m_{2}=8$ & $m_{3}=2$ \\
Inertia $\left(\mathrm{kgm}^{2}\right)$ & $I_{1}=50$ & $I_{2}=50$ & $I_{3}=50$ \\
link distance $(\mathrm{m})$ & $l_{c_{1}}=0.8$ & $I_{c_{2}}=0.65$ & $l_{c_{3}}=0.35$ \\
\hline \hline
\end{tabular}

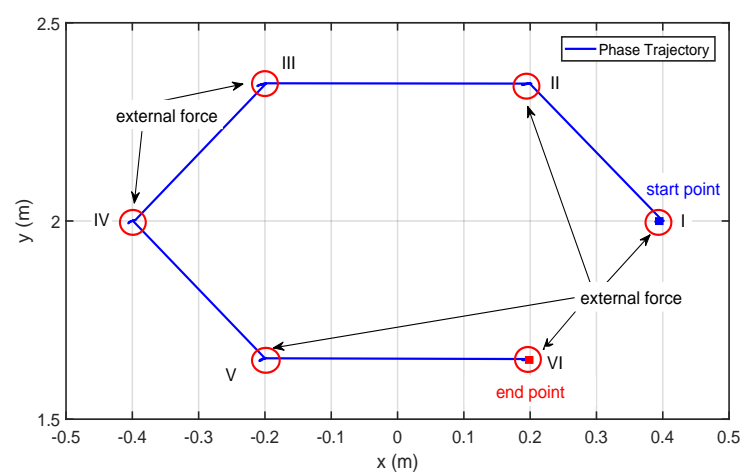

Fig. 4. Phase trajectory of the robot control

where the manipulator inertial matrix $M(q)$ and Coriolis matrix $C(q, \dot{q})$ are described as

$$
\begin{aligned}
M(q) & =\left[\begin{array}{ccc}
M_{11} & * & * \\
M_{21} & M_{22} & * \\
M_{31} & M_{32} & M_{33}
\end{array}\right] \\
C(q, \dot{q}) & =\left[\begin{array}{ccc}
C_{11} & C_{12} & C_{13} \\
C_{21} & C_{22} & C_{23} \\
C_{31} & C_{32} & C_{33}
\end{array}\right] \quad G(q)=\left[\begin{array}{l}
G_{11} \\
G_{21} \\
G_{31}
\end{array}\right]
\end{aligned}
$$

The robot are commanded to reach 6 set points around a circle in a sequence as shown in Fig.4. As see from the figure, when the robot has reached one set point, an external force will applied on the robot and the robot will hold on the target point with 2 seconds. Then the robot moves to the next set point. The initial position of the manipulated is set to $x(0)=[0.4,2]$, and the velocity is chosen to $\dot{x}(0)=[0,0]$. The control gains are selected to be positive definite matrix as $K_{p}=\operatorname{diag}\{50,50,50\}$, and $K_{1}=\operatorname{diag}\{10,10,10\}$. The gain of the disturbance observer is chosen to $\Gamma=\operatorname{diag}\{0.1,0.1,0.1\}$. The external force is chosen to $\tau_{d}=[-10,-20,10]$.

An overlook of the tracking performance is shown in Fig.4, and we can see that the robot has successfully reached every target point. To clearly shown the advantage of the proposed method, the tracking performance of robot holding the target point in each stage is depicted as shown in Figs.5-7. The tracking errors in $x$ direction and $y$ direction are depicted in Figs.5 and 6. We can see that, although external forces are applied, the robot is able to hold on the target point with only a slight movement. Moreover, the tracking errors remain around zeros such that the prescribed bounded are not violated. In comparison, an obvious shift of the robot manipulator has been observed under the PID controller when 


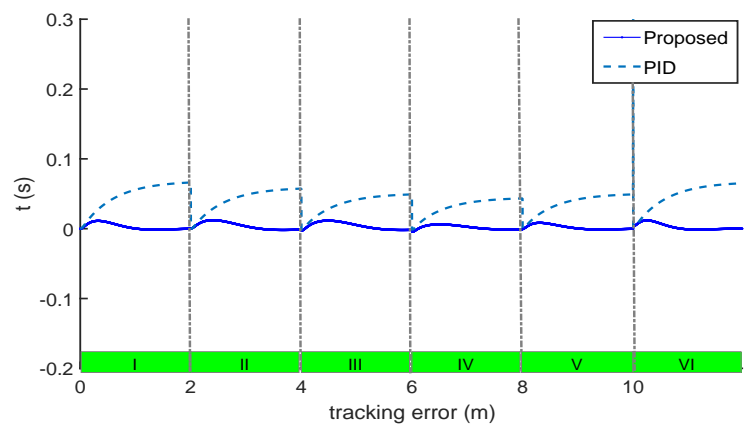

Fig. 5. Tracking error in $x$ direction

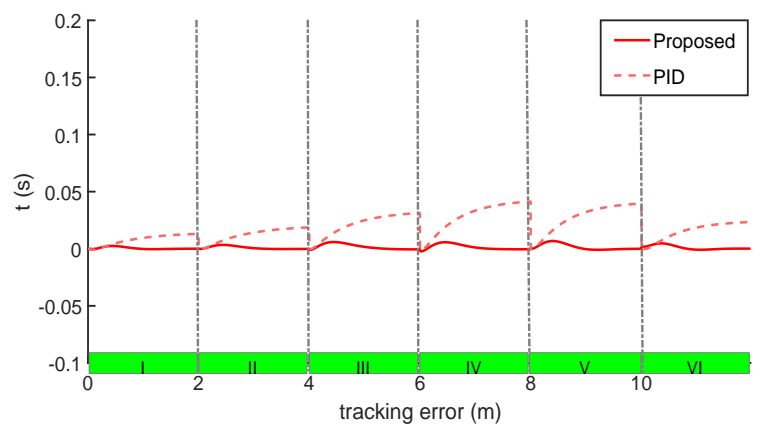

Fig. 6. Tracking error in $y$ direction

the external force is employed. The profile of control input is depicted in Fig.7. We can see that the control inputs are able to adjust in terms the external forces rapidly and the robot is able to hold on the desired set point effectively. Thus, the effectiveness of the proposed controller has been demonstrated.

\section{CONCLUSION}

Based on a null space control framework and a prescribed performance control law, this paper develops a robot controller to ensure both the transient tracking control performance and the compliant motion control behaviour of the redundant manipulator. The prescribed transient tracking performance is guaranteed by using an error transformation based task space controller. Additionally, the 1 behaviour is achieved by using the null space dynamics control algorithm with an adaptive disturbance observer, which is able to estimate and cancel the effect of the external torque and to reduce the task tracking error. The closed-loop robot system is proved to be asymptotic stable using the Lyapunov theorem and Lasalle's invariance principle. Simulations based on a 3DOF were carried out to demonstrate the effectiveness of the proposed controller.

\section{ACKNOWLEDGMENT}

This work was supported in part by the Nature Science Foundation of China under Grant No. 61473120, 51575412, 51575338 and 51575407, the EU Seventh Framework Programme (FP7)-ICT under Grant No. 611391, the Science and Technology Planning Project of Guangzhou under Grant

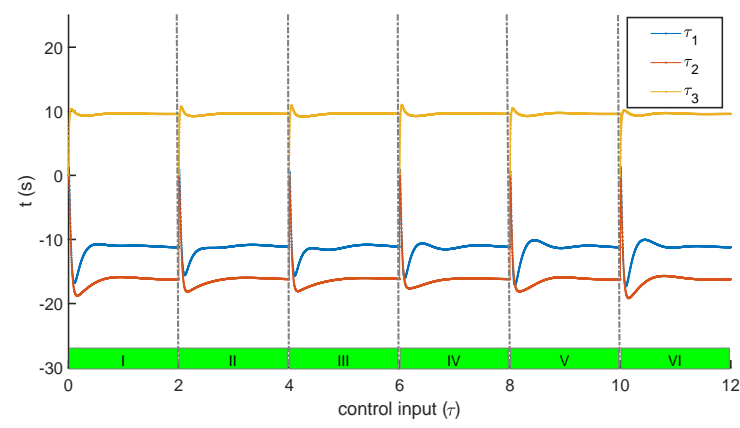

Fig. 7. Control input of the robot manipulator

No. 201607010006, the State Key Laboratory of Robotics and System under Grant No. SKLRS-2017-KF-13, the Fundamental Research Funds for the Central Universities under Grant No. 2017ZD057, and the Research Project of State Key Lab of Digital Manufacturing Equipment and Technology of China under Grant No. DMETKF2017003.

\section{REFERENCES}

[1] A. Bicchi, G. Tonietti, M. Bavaro, and M. Piccigallo, "Variable stiffness actuators for fast and safe motion control," in the Proceedings of the Eleventh International Symposium on Robotics Research. 2005, pp. $527-536$.

[2] C. Yang, H. Ma, and M. Fu, Advanced Technologies in Modern Robotic Applications. Springer, 2016.

[3] C. Yang, Y. Jiang, Z. Li, W. He, and C.-Y. Su, "Neural control of bimanual robots with guaranteed global stability and motion precision," IEEE Transactions on Industrial Informatics, vol. 13, no. 03, pp. 1162$1171,2017$.

[4] D. Guo and Y. Zhang, "Acceleration-level inequality-based man scheme for obstacle avoidance of redundant robot manipulators," IEEE Transactions on Industrial Electronics, vol. 61, no. 12, pp. 6903-6914, Dec 2014.

[5] B. Siciliano and O. Khatib, Springer Handbook of Robotics. Springer, 2016.

[6] H. Sadeghian, L. Villani, M. Keshmiri, and B. Siciliano, "Taskspace control of robot manipulators with null-space compliance," IEEE Transactions on Robotics, vol. 30, no. 2, pp. 493-506, 2014.

[7] A. Albu-Schäffer, C. Ott, and G. Hirzinger, "A unified passivitybased control framework for position, torque and impedance control of flexible joint robots," The International Journal of Robotics Research, vol. 26, no. 1, pp. 23-39, 2007.

[8] C. Ott, A. Dietrich, and A. Albu-Schäffer, "Prioritized multi-task compliance control of redundant manipulators," Automatica, vol. 53, pp. 416-423, 2015.

[9] H. Sadeghian, L. Villani, M. Keshmiri, and B. Siciliano, "Multi-priority control in redundant robotic systems," in IEEE/RSJ International Conference on Intelligent Robots and Systems (IROS). 2011, pp. 37523757.

[10] F. Vigoriti, F. Ruggiero, V. Lippiello, and L. Villani, "Control of redundant robot arms with null-space compliance and singularity-free orientation representation," Robotics and Autonomous Systems, vol. 100, pp. 186-193, 2018.

[11] C. Yang, K. Huang, H. Cheng, Y. Li, and C.-Y. Su, "Haptic identification by elm-controlled uncertain manipulator," IEEE Transactions on Systems, Man, and Cybernetics: Systems, 2017.

[12] L. Cheng, Z.-G. Hou, M. Tan, and W. J. Zhang, "Tracking control of a closed-chain five-bar robot with two degrees of freedom by integration of an approximation-based approach and mechanical design," IEEE Transactions on Systems, Man, and Cybernetics, Part B: Cybernetics, vol. 42, no. 5, pp. 1470-1479, 2012.

[13] K. M. Lynch and T. Murfey, Control of Nonprehensile Manipulation. Springer, 2002

[14] Y. Oh, W. Chung, and Y. Youm, "Extended impedance control of redundant manipulators based on weighted decomposition of joint space," Journal of Field Robotics, vol. 15, no. 5, pp. 231-258, 1998. 
[15] O. Khatib, "Inertial properties in robotic manipulation: An object-level framework," The International Journal of Robotics Research, vol. 14, no. 1, pp. 19-36, 1995.

[16] W. H. Chen, "Nonlinear disturbance observer-enhanced dynamic inversion control of missiles," Journal of Guidance, Control, and Dynamics, vol. 26, no. 1, pp. 161-166, 2003.

[17] J. P. LaSalle, The Stability and Control of Discrete Processes. Springer, 2012.

[18] C. R. Carnigan, "Adaptive tracking for complex systems using reducedorder models," in the Proceedings of IEEE International Conference on Robotics and Automation. 1990, pp. 2078-2083 